\title{
Evaluation of cardiovascular sympathetic reactivity in Normotensive offspring of Hypertensive parents
}

\author{
Devaki .P.R. ${ }^{1}$, Saikumar.P ${ }^{2}$, K.Anushri ${ }^{3}$ \\ ${ }^{I}$ Associate Professor, Dept of physiology, Sree Balaji Medical College and Hospital, Chromepet, Bharath \\ University, Chennai,INDIA \\ 2Professor \& H.O.D, Dept of physiology, Sree Balaji Medical College and Hospital, Chromepet, Bharath \\ University, Chennai,INDIA \\ ${ }^{3}$ II M.B.B.S Student, Dept of physiology,Sree Balaji Medical College and Hospital,Chromepet, Bharath \\ University, Chennai,INDIA
}

\begin{abstract}
Background: Sympathetic reactivity is defined as an exaggerated response to stress. Studies have shown that enhanced sympathetic response to stress tilts the balance towards Sympathetic over activity which in turn indicates a pre hypertensive state. Genetic predisposition definitely plays an important role in the development of hypertension. Hence, there is a need to explore the Sympathetic reactivity in children of hypertensive parents.

Aim \& Objective: The aim of this study is to assess the cardiovascular response to sympathetic activity in Normotensive off spring of Hypertensive parents $(\mathrm{NH})$ and compare the same with Normotensive off spring of Normotensive parents $(N N)$.

Materials and Methodology: 60 children of age group 18-22 years, both males and females with normal BMI were included in the study. Based on the questionnaire they were grouped as Normotensive children of Hypertensive parents $(\mathrm{NH})$ and Normotensive children of Normotensive parents (NN). Heart rate and Blood pressure at rest and during isometric hand grip exercise and mental stress test was measured. Statistical analysis was done to compare the blood pressure and heart rate at rest and during isometric hand grip exercise and mental stress test between both the groups using nonparametric test.

Results: A statistically significant increase in diastolic blood pressure was observed in offspring of hypertensive parents when compared to the offspring of normotensive parents indicating sympathetic overactivity .
\end{abstract}

Keywords-Isometric hand grip, Mental stress test, Sympathetic reactivity.

\section{Introduction}

Essential hypertension is considered as a multifaceted progressive disease which spans over several decades of life. Studies have shown that if either of the parent is hypertensive than there is $25 \%$ chance that the children may become hypertensive and the chance is $50 \%$ when both the parents are hypertensives ${ }^{1,2}$. Controversial results have been reported as to there may or may not be an exaggerated sympathetic response to stress in normotensive offspring of hypertensive parents.In a study done by Hastrup et al they observed higher reactivity in normotensive male children of hypertensive parents but females were not included in this study ${ }^{3}$. Greater increase in total peripheral resistance was noted in normotensive children of parents who were hypertensive in a study done by Devisser et al .However, the subjects of this study were of a wider age ranging from 8-33 years4.Gerin and Pickering found no difference in the initial reactivity between normotensive children of hypertensive parents and normotensive parents but this study involves multiple ethnicity ${ }^{5}$. Though studies have shown that genetic factor plays an important role in the development of hypertension, the role of other factors like age, gender and ethnicity could not be neglected .Hence, in our study we have included subjects with homogeneity in regard to age ,BMI, base line blood pressure and race.

Study population includes:

\section{Materials And Methodology}

- Normotensive offsprings of normotensive parents $(\mathrm{NN}) \mathrm{n}=29$

- Normotensive offsprings of hypertensive parents $(\mathrm{NH}) \mathrm{n}=31$

- Both the gender (males- 28,females-32).

- Age group-18-21years.

- Normal BMI 


\section{Exclusion criteria:}

- Smokers,

- Children who do regular exercise,

- Children of diabetic parents

- Comorbid condition affecting sympathetic nervous system activity.

- Taking drugs interfering with ANS activity .

Study protocol: Study was approved by the institutional ethical committee of Sree Balaji Medical College and Hospital. Proper written informed consent was obtained from all the participants. Subjects were asked to abstain themselves from caffeinated drinks 16 hours before testing.Tests were carried out in the morning between 8-11 AM, two hours after a standardized meal in the Research lab of the Department of Physiology , Sree Balaji Medical College and Hospital. Before testing subjects were asked to empty their bladder . The test was carried out by the same observer who was blinded about the group to which the subject belongs to in order to avoid the bias .

Experimental protocol: Heart rate and Blood pressure were recorded using Non Invasive Blood Pressure (NIBP) monitor (PLANET -50) in all the sixty subjects at rest, during isometric hand grip test and mental arithmetic task .

Isometric hand grip test: Maximum voluntary contraction was determined by asking the subject to press the hand grip dynamometer thrice with maximum effort. Subjects were asked to maintain $30 \%$ of the average maximum voluntary contraction (MVC) for 2 minutes. Subjects were instructed to count softly to avoid alteration in respiratory pattern ${ }^{6.8}$.

Mental Arithmetic Task: Subjects were asked to perform sequential subtraction of one to two integers from three to four digit integers. The test was carried out for three rounds. Each round lasted for 2 minutes . The subject was asked to say the answer aloud ${ }^{7.8}$. At the end of the task the difficulty index was marked in the Likert scale. It was ensured that there is uniformity in the difficulty index.

\section{Results}

Results were analyzed by non-parametric $t$ test Mann whitney $U$ for independent samples using spss software version 11. The base line characteristics were comparable between NH and NN(TABLE-1). A statistically significant increase in diastolic blood pressure was observed in NH group as compared to the NN group during isometric hand grip test and mental arithmetic task as shown by the TABLE $2 \& 3$.

\section{Table-1- Comparison of baseline characteristics between NN and NH}

\begin{tabular}{|l|l|l|}
\hline BASELINE CHARACTERS & NN & NH \\
\hline AGE ( YEARS) & $19.7 \pm 1.07$ & $20.1 \pm 0.8$ \\
\hline BMI ( kg/meter square) & $21.7 \pm 1.52$ & $21.5 \pm 1.6$ \\
\hline SYSTOLIC BP (mm Hg) & $113.3 \pm 9.3$ & $116.6 \pm 8.09$ \\
\hline DIASTOLIC BP(mm Hg) & $\mathbf{7 4 . 1} \pm 5.2$ & $74.9 \pm 7.3$ \\
\hline $\begin{array}{l}\text { HEART RATE ( beats per } \\
\text { minute ) }\end{array}$ & $\mathbf{8 4 . 4} \pm 7.3$ & $82 \pm 8.6$ \\
\hline
\end{tabular}

Table -2-Comparison of the rise in the Blood Pressure And Heart Rate from the Resting value During Isometric Hand Grip Test between NN and NH

\begin{tabular}{|l|l|l|l|}
\hline & SBP $(\mathrm{mmHg})$ & DBP(mmHg) & HR beats/min \\
\hline NN & $9.27 \pm 7.4$ & $8.06 \pm 5.8$ & $8.48 \pm 6.9$ \\
\hline NH & $14.1 \pm 10.5$ & $14.03 \pm 8.2 *$ & $11.7 \pm 8.6$ \\
\hline
\end{tabular}


Table -3-Comparison of the rise in the Blood Pressure And Heart Rate from theResting value during Mental Arithmetic Test between NN and NH

\begin{tabular}{|l|l|l|l|}
\hline & SBP $\mathrm{mmHg}$ & DBP $\mathrm{mmHg}$ & HR beats/min \\
\hline NN & $\mathbf{6 . 8 6} \pm \mathbf{8 . 5}$ & $\mathbf{3 . 3 4} \pm \mathbf{4 . 2}$ & $\mathbf{7} \cdot 17 \pm \mathbf{8 . 9 5}$ \\
\hline NH & $9.16 \pm 7.31$ & $6.19 \pm 5.13^{*}$ & $8.77 \pm 7.59$ \\
\hline
\end{tabular}

* p Value - $<0.05$-Statistically Significant

\section{Discussion}

It is a well known fact that genetic factors play an important role in the etiology of human hypertension however its role remain undefined. This possibly could be due to the variety of confounding factors including the heterogeneity of the populations studied age, gender, lifestyle, and environment. In this study it was observed that the resting parameters were comparable between both the groups however a statistically significant increase in the diastolic blood pressure value from the rest was observed in children of hypertensive parents . A rise in blood pressure response to stress at young age may be an early predictor for the subject to become hypertensive in future.

An increase in the diastolic blood pressure response to isometric hand grip and mental arithmetic test may be due to Sympathetic Nervous System activation which by its vasoconstrictor effects mediates the stress induced increase in blood pressure that may promote the development and progression of the hypertension .Though ill defined, genetic factors may play a role in blood pressure control, in adrenergic modulation of cardiovascular drive, as well as in multiorgan response to stress .

In a study done by Guido et al an increased sympathetic nervous system activity during stress was observed In Phosducin (pdc) gene knocked out mice .Phosducin is a 33-kDa cytosolic regulator of G proteinmediated signaling .From This Observation They Suggested That Phosducin Plays An Important Role In The Genetic Determination Of Hypertension ${ }^{9}$. The Possible Role Of Phosducin Gene Knock Out In The Exaggerated Sympathetic Response Observed In Children Of Hypertensive Parents Needs To Be Explored. In A Study Done By Zhou Et Al Genetic Variations In Neuropeptide Y Receptors Was Observed And It Appears To Be Associated With High Blood Pressure ${ }^{10}$. Seratonin Transporter Gene Polymorphism Has Shown To Increase The Cardiovascular Reactivity To Mental Stress ${ }^{11}$ Suggesting That The Gene Responsible For Stress Reactivity In Case Of Different Types Of Stress May Not Be The Same.

\section{Conclusion}

Hence it may be concluded that the cardiovascular sympathetic reactivity was exaggerated in offspring of hypertensive parents when compared to offspring of normotensive parents. Any one of the genetic variation linked to sympathetic abnormality may be the probable reason for the increased cardiovascular response to stress identified in offspring of hypertensive parents. The exaggerated response to stress may be an early marker in determining which person will eventually develop hypertension. Studies involving identification of genetic component involved in the exaggerated stress response may be helpful in identifying the underlying genetic cause.

\section{References}

[1]. Olson RP, Kroon JS. Biobehavioral treatment of_essential hypertension. In: Schwartz MS (ed). Biofeedback:A Practitioner's Guide. The Guilford Press: NewYork, 1987, pp 316-339.

[2]. Julius S,Weder AB, Egan BM. Pathophysiology of earlyhypertension: implication for epidemiologic research.In: Gross F, Strasser T (eds). Mild Hypertension: RecentAdvances. Raven Press: New York, 1983, pp 219-236.

[3]. Hastrup Jl,light KC ,Obrist PA, Parental response and cardiovascular response to stress in healthy young adults .Psychophysiology $1982,19,615-623$.

[4]. De Visser DC et al,Cardiovascular response to mental stress in offsprings of hypertensive parents : the Dutch hypertension and offspring study.J Hypertens $1995 ; 13: 901-908$.

[5]. Gerin W ,Pickering GT, Association between delayed recovery of blood pressure after acute mental stress and parental history of hypertension.J Hypertens 1995 ; 13: 603-610.

[6]. Wasmund Wl, Westerholm EC, Watenpaugh DE, Stephen I, et al. The interactive effects of the mental and physical stress on the cardiovascular control. J Appl Physiol 2002; 92: 1828-34.

[7]. Karen A Matthews, Karen L Woodall, Michael T. The cardiovascular reactivity to stress predicts the future blood pressure status. Hypertension. 1993,22:479-85.

[8]. Devaki Perumal Rajaram, R.Karthikeyan, P.Saikumar et al. The Cardiovascular response to the acute physical and mental stress in Type -2 Diabetes Mellitus .J of clinical and diagnostic research. September2012(suppl)vol-6(7):1237-1240.

[9]. Guido Grassi et al, Phosducin — a candidate gene for stress-dependent hypertension. J Clin Invest. 2009;119(12):3515-3518.

[10]. Zhou, Z., et al. 2008. Genetic variation in human NPY expression affects stress response and emotion. Nature. 452:997-1001.

[11]. Redford B. Williams, MD et al. Childhood Socioeconomic Status and Serotonin Transporter Gene Polymorphism Enhance Cardiovascular Reactivity to Mental Stress. Psychosomatic Medicine January 2008 vol. 70 no. 1 32-39. 